Biomacromolecules. Author manuscript; available in PMC 2017 July 04.

Published in final edited form as:

Biomacromolecules. 2016 June 13; 17(6): 2248-2252. doi:10.1021/acs.biomac.6b00481.

\title{
Adhesion of blood clots can be enhanced when copolymerized with a macromer that is cross-linked by coagulation factor XIIla
}

\author{
Karen Y.T. Chan ${ }^{1}$, Chunyi Zhao ${ }^{1}$, Erika M.J. Siren², Jeanne C.Y. Chan ${ }^{1}$, Jeffrey Boschman ${ }^{1}$, \\ and Christian J. Kastrup ${ }^{1, *}$ \\ ${ }^{1}$ Michael Smith Laboratories and Department of Biochemistry and Molecular Biology, University of \\ British Columbia, Vancouver, BC, Canada \\ ${ }^{2}$ Centre for Blood Research, Department of Pathology and Laboratory Medicine, and the \\ Department of Chemistry, University of British Columbia, Vancouver, BC, Canada
}

\section{Abstract}

The adhesion of blood clots to blood vessels, such as through the adhesion of fibrin, is essential in hemostasis. While numerous strategies for initiating clot formation and preventing clot lysis are being developed to create improved hemostatic agents, strategies for enhancing clot adhesion have not been widely explored. Here, we show that adhesion of blood clots can be increased by adding a previously characterized synthetic polymer that is cross-linked by coagulation factor XIIIa during clotting. Addition of the polymer to normal plasma increased the adhesive strength of clots by 2 -fold, and recovered the adhesive strength of non-adhesive fibrinogen-deficient whole blood clots to $1.9 \pm 0.14 \mathrm{kPa}$, which is similar to the adhesive strength of a fibrinogen-rich clot (1.8 $\pm 0.64 \mathrm{kPa}$ ). The polymer also enabled plasma clots to remain adhered under fibrinolytic conditions. Demonstrating that the adhesive strength of clots can be increased with a synthetic material provides a potential strategy for creating advanced hemostatic materials, such as treatments for fibrinogen deficiency in trauma-induced coagulopathy.

\section{Graphical Abstract}

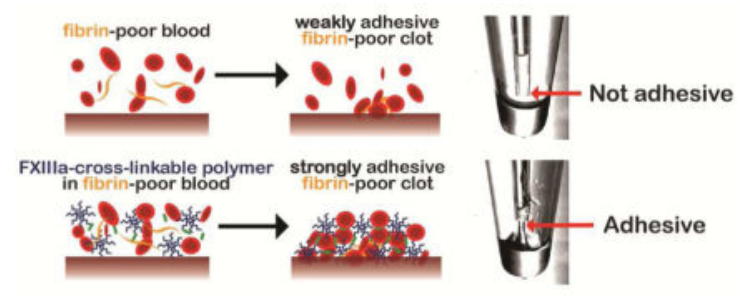

"Corresponding Author: ckastrup@msl.ubc.ca. Author Contributions

The manuscript was written through contributions of all authors. All authors have given approval to the final version of the manuscript. The authors declare no competing financial interest.

Supporting Information. Detailed methods describing the GPC and ${ }^{1} \mathrm{H}$ NMR experiments, and a reproduction of the ${ }^{1} \mathrm{H}$ NMR spectra and its analysis, are included. This material is available free of charge via the Internet at http://pubs.acs.org. 


\section{Keywords}

biomaterials; transglutaminase; polyethylene glycol; hemostasis; fibrinogen deficiency; composite clot

\section{INTRODUCTION}

Blood clots must adhere to vasculature to stop bleeding effectively. Multiple components of clots, including platelets and fibrin, have adhesive properties that help seal damaged vessels ${ }^{1}$. Strongly-adhered clots are also less likely to detach from the inside of vessels, where they would otherwise flow downstream and, in some cases, embolize smaller vessels ${ }^{2}$. Strategies for enhancing the adhesive strength of clots, particularly when endogenous adhesive components are depleted, may increase the efficacy of existing hemostatic agents, but this has not been widely explored.

Many natural and synthetic tissue adhesives have been developed to close wounds ${ }^{3}$. Fibrin sealants in particular are effective for surgical applications when applied topically ${ }^{4}$. The adhesive strength and hemostatic potential of topical fibrin sealants increase with the concentration of fibrinogen ${ }^{4}$. Endogenous clots are also better at achieving hemostasis when fibrinogen is transfused into the blood stream ${ }^{5}$; fibrinogen replacement therapy has been used both as a prophylactic and acute treatment of bleeding in patients with congenital and acquired fibrinogen deficiency ${ }^{6-8}$. This suggests that the natural adhesion of endogenous clots can be increased by adding exogenous fibrinogen; however, there are downsides to this strategy. Fibrin is susceptible to fibrinolysis, which accompanies rebleeding 9 . Furthermore, both recombinant and isolated fibrinogen are expensive to produce, with isolated fibrinogen requiring additional steps to mitigate the risk of blood-borne pathogens ${ }^{3,4,7}$. Transfusions of blood products are also prohibited by some religions ${ }^{10}$. Together, this provides motivation to identify alternative strategies for increasing the adhesion of endogenous clots.

Fibrin is a component of blood clots produced by thrombin-mediated cleavage of its zymogen, fibrinogen, in response to vascular injury. It functions by self-assembling into insoluble fibers, which are cross-linked by coagulation factor XIIIa (FXIIIa) to stabilize and adhere clots to tissue ${ }^{4}$. FXIIIa is a transglutaminase that forms covalent bonds between certain glutamine- and amine-containing molecules ${ }^{4,11}$. It is highly promiscuous and can covalently link fibrin to many substrates, including collagen, fibronectin, and platelets surface proteins ${ }^{11-13}$. Several synthetic FXIIIa-cross-linkable polymers have been developed, including a well-characterized 8-armed polyethylene glycol conjugated to a glutamine-containing peptide derived from a2-antiplasmin (Q-PEG) ${ }^{14-19}$. Q-PEG can be cross-linked by FXIIIa to many polyamine substrates, such as spermidine ${ }^{14-19}$. This FXIIIacross-linkable polymer (Q-PEG and spermidine) can be gelled by the coagulation network when added to fibrinogen-deficient (FG-def) plasma $^{19}$. We hypothesized that copolymerizing blood clots with this polymer would increase the clot's adhesive strength and also restore adhesion of fibrin-poor blood clots, such as clots formed during fibrinogendepletion or clots exposed to fibrinolytic enzymes. 
Adding polymers to fibrin clots is known to increase their mechanical strength by contributing mechanical impedance to the movement of the fibrin network ${ }^{20,21}$. This does not necessarily increase the adhesion of the material, and no quantitative study on increasing clot adhesion by adding a synthetic polymer has been reported. Here, we show that the lap shear strength of blood clots supplemented with a FXIIIa-cross-linkable polymer increased in a wide-range of conditions in a FXIIIa-dependent manner. The polymer also restored the adhesive strength of whole blood clots that were fibrin-poor.

\section{MATERIALS AND METHODS}

\section{Synthesizing Q-PEG}

Q-PEG was synthesized as previously described ${ }^{19}$. In brief, a peptide derived from a2antiplasmin, acetyl-NQEQVSPLTLLKKGC (Thermo Fisher Scientific), was conjugated to 8-armed PEG maleimide (40kDa, Creative PEGWorks) through the peptide's cysteine thiol. Q-PEG was purified by dialyzing first in Tris-EDTA buffer, then in water. Further purification was achieved using a desalting column (Thermo Fisher Scientific). The purified conjugates were concentrated by a Centri-prep filter (Amicon®Ultra 10K, Millipore), lyophilized, and stored at $-20^{\circ} \mathrm{C}$ until use. The molecular weight of Q-PEG was determined by gel permeation chromatography (GPC) and the degree of functionalization was determined by ${ }^{1} \mathrm{H}$ NMR analysis (see Supplementary Information for detailed methods). On average, 4 of the 8 PEG maleimide arms were functionalized with the target peptide (Figure $\mathrm{S} 1)$. The remaining maleimide groups were reacted with 2-mercaptoethanol.

\section{Preparing FG-def human and mouse whole blood}

This study was approved by the University of British Columbia research ethics boards. Informed consent was obtained from all healthy human volunteers prior to whole blood donation. Human whole blood was collected into Vacutainer ${ }^{\mathrm{TM}}$ tubes containing K3 EDTA ( $15 \%$ solution) or sodium citrate $(0.105 \mathrm{M})$ (BD Biosciences), and centrifuged at $2000 \mathrm{~g}$ for 15 minutes to pellet the blood cells ${ }^{22}$. The plasma was discarded and blood cells were washed thrice with citrated FG-def human plasma $(0.06 \mathrm{mg} / \mathrm{mL}$ fibrinogen, Affinity Biologicals) to deplete the fibrinogen. The washed blood cells were resuspended in citrated FG-def human plasma to prepare FG-def human whole blood. To obtain FG-def mouse whole blood, 50\% 129SvJ/50\% C57Bl/6 animals with a complete FG- chain knockout were used $^{23}$. Whole blood was collected via cardiac puncture and citrated $(7 \mathrm{mM})$.

\section{Copolymerizing Q-PEG with blood clots and measuring their adhesion}

Whole blood or plasma was diluted to a solution yielding $75 \%$ blood or plasma and containing final concentrations of Q-PEG $(46 \mathrm{mg} / \mathrm{mL})$, spermidine $(1.3 \mathrm{mM}$, SigmaAldrich), $\mathrm{CaCl}_{2}(50 \mathrm{mM})$, purified human FXIII zymogen $(0.067 \mathrm{mg} / \mathrm{mL}$, Haematologic Technologies Inc.), and silica particulates/APTT activator (2.5\% v/v, Pacific Hemostasis). In experiments where FXIIIa was inhibited, T101 (FXIIIa inhibitor, $3.8 \mathrm{mM}, 1,3,4,5$ Tetramethyl-2-[(2-oxopropyl)thio]imidazolium chloride, ZEDIRA $\mathrm{GmbH}$ ) was added to the reaction mix. In experiments with excised sheep vessels, the common aortae were collected from Dorset cross sheep and endothelial layers were removed to expose the subendothelia. A higher concentration of FXIII $(0.13 \mathrm{mg} / \mathrm{mL})$, and bovine thrombin $(24 \mathrm{U} / \mathrm{mL}$, Sigma- 
Aldrich) was used to activate clotting. Blue food coloring ( $0.5 \% \mathrm{v} / \mathrm{v}$, Club House) was added to visualize the blood plasma between the translucent blood vessels. Adhesion was measured with a TA Q800 dynamic mechanical analyzer equipped with a shear-sandwich clamp (TA Instruments). Collagen-coated glass coverslips or excised sheep vessels were attached to clamps using UV-curable glue (Loon Outdoors). Q-PEG-containing blood was loaded onto a $7 \times 7 \mathrm{~mm}$ section that was $0.46 \mathrm{~mm}$ thick between the coverslips or excised vessels, and the edges were sealed with fluorocarbon grease (Krytox grease, DuPont) to prevent evaporation. Clots were formed for 90 or $180 \mathrm{~min}$ on collagen or vessels respectively to allow the clots to be fully cross-linked by FXIIIa. A force ramp was applied at $0.01 \mathrm{~N} / \mathrm{min}$ until the clot failed. The normality of data sets was verified with a Shapiro-Wilk test and $\mathrm{p}$ values were calculated by Welch's analysis of variance to account for unequal sample sizes and variances $^{24}$.

\section{Measuring the gelation of Q-PEG and collagen in FG-def plasma}

In experiments investigating the interaction between Q-PEG and collagen, FG-def plasma (1 $\mathrm{Qg} / \mathrm{mL}$ fibrinogen, Affinity Biologicals) was used. The reaction mix contained FG-def plasma (14\% v/v), Q-PEG (26 mg/mL), rat-tail collagen I ( $4.1 \mathrm{mg} / \mathrm{mL}$, Sigma-Aldrich), $\mathrm{CaCl}_{2}(30 \mathrm{mM})$, purified human FXIII $(0.1 \mathrm{mg} / \mathrm{mL})$, and bovine thrombin $(69 \mathrm{U} / \mathrm{mL})$. T101 $(6.9 \mathrm{mM})$ was added in specified samples to inhibit FXIIIa. Green fluorescent beads $\left(6 \times 10^{8}\right.$ particles $/ \mathrm{mL}$ ) were added to the reaction mix to monitor gelation by fluorescence microscopy as previously described ${ }^{19}$.

\section{Measuring the lysis of Q-PEG copolymerized clots in normal plasma}

Plasma was diluted (58\%) in a solution yielding final concentrations of Q-PEG (34 mg/mL), spermidine $(1 \mathrm{mM}), \mathrm{CaCl}_{2}(39 \mathrm{mM})$, and purified human FXIII zymogen $(0.07 \mathrm{mg} / \mathrm{mL})$. Ampicillin (1.9 mg/ml, Sigma-Aldrich) was added to the reaction mix to prevent bacterialinduced clot lysis. Tissue plasminogen activator (tPA, $15 \mathrm{nM}$, Genentech), a fibrinolytic enzyme, and bovine thrombin $(19 \mathrm{U} / \mathrm{mL})$, an activator of clotting, were then added. Clot formation occurred immediately after thrombin was added ( $<10 \mathrm{~min})$ and shear adhesive strength of the resulting clot on collagen-coated coverslips was measured 90 min later. Clot lysis times were detected by fluorescence microscopy via the Brownian movement of green fluorescent beads $\left(6 \times 10^{8}\right.$ particles $\left./ \mathrm{mL}\right)$ in the reaction mixture.

\section{RESULTS AND DISCUSSION}

\section{Adhesion of normal plasma clots can be increased with a synthetic polymer}

To test if adding a FXIIIa-cross-linkable polymer to plasma could increase the adhesion of the copolymerized clot, Q-PEG, spermidine, and additional FXIII zymogen were mixed with human plasma and clotted on collagen-coated coverslips or excised blood vessels (Figure 1a). Collagen is a major component of the sub-endothelial layers of blood vessels ${ }^{25}$. The adhesive strength of the clot to the collagen-coated surface was measured, and detachment from the substrate was verified to ensure adhesive failure of the material. The composite clot, containing supplementary FXIII zymogen, Q-PEG, and spermidine, was over 4-fold more adhesive than a normal plasma clot, and over 2-fold more adhesive than a control clot supplemented with the same amount of FXIII (Figure 1b). This increase in adhesive strength 
required both Q-PEG and spermidine, demonstrating that a FXIIIa-cross-linkable polymer can be used to increase the adhesion of a fibrin clot. To test if the increased adhesion to collagen translates to increased adhesion to blood vessels, the adhesion of the composite clot to sheep aorta was tested ex vivo. Composite clots containing Q-PEG and spermidine were formed on the exposed subendothelia of excised sheep arteries and their adhesive strengths were measured (Figure 1c). The adhesive strength of the composite clot was roughly 2 -fold higher than a clot supplemented with FXIII alone, showing clot adhesion to excised vessels can be increased as well.

\section{Increased adhesion is dependent on FXIIla}

To determine the mechanism of increased adhesion to collagen, we investigated whether collagen and Q-PEG bind to each other in a FXIIIa-dependent manner. Collagen is a known amine-donor for FXIIIa ${ }^{12}$. To test if Q-PEG and collagen bind to each other in plasma, their ability to form a gel together was measured (Figure 2a-b). They were added to plasma deficient in fibrinogen and free of spermidine, because FXIIIa is known to cross-link both of these amine donors to Q-PEG. Without these amine donors or collagen, Q-PEG did not gel. When collagen was added, gelation with Q-PEG did occur, except when T101, an irreversible inhibitor of FXIIIa, was added. This showed that FXIIIa was responsible for binding Q-PEG to collagen, either directly or indirectly through other FXIIIa substrates present in plasma. To test if the Q-PEG and spermidine components of composite clots were also bound to collagen-coated surfaces by FXIIIa activity, their lap-shear strength was measured in FG-def plasma (Figure 2c). The adhesive shear strength of FG-def plasma alone to collagen was below detection, whereas it was $1.8 \pm 0.27 \mathrm{kPa}$ when Q-PEG and spermidine were added, similar to clots of normal plasma $(2.0 \pm 0.85 \mathrm{kPa}$, shown in Figure 1b). Adhesion was dependent on the activity of FXIIIa, as the shear strength dropped below detection when T101 was added. Together, these results are consistent with a mechanism where increased adhesion of clots containing the FXIIIa-cross-linkable polymer was due to Q-PEG binding to collagen by the transglutaminase activity of FXIIIa.

\section{The adhesive strength of fibrinogen-depleted clots can be restored to that of normal whole-blood clots}

We next tested whether the adhesion of whole blood (WB) clots and fibrinogen-deficient whole blood (FG-def WB) clots could be increased by addition of Q-PEG (Figure 3a). We were interested in the adhesion of FG-def WB clots because depletion of fibrinogen is a major cause of uncontrolled bleeding during trauma, which may potentially be addressed by an agent that enhances adhesion ${ }^{26}$. Human WB clots supplemented with Q-PEG, spermidine and FXIII zymogen did not have adhesive strengths higher than those of human WB clots with supplemental FXIII alone (Figure 3b). Human FG-def WB clots were non-adhesive, but adhesion was recovered when Q-PEG and spermidine were added (Figure 3c). Remarkably, addition of the synthetic polymer increased the shear strength of FG-def WB clots to those of normal WB clots ( $1.9 \mathrm{kPa} \pm 0.14$ and $1.8 \pm 0.64 \mathrm{kPa}$, respectively). Similarly, blood clots from mice with congenital fibrinogen-deficiency were non-adhesive, but the addition of QPEG and spermidine recovered adhesion to $0.47 \pm 0.20 \mathrm{kPa}$ (Figure 3d). 


\section{Clot detachment during fibrinolysis can be inhibited by adding a FXIIla-cross-linkable polymer}

To test if the FXIIIa-cross-linkable polymer can also increase the adhesive strength of clots compromised by fibrinolysis ${ }^{27}$, tPA was added to normal plasma with and without Q-PEG and spermidine. The lysis time of normal plasma was substantially extended by adding QPEG and spermidine, increasing from $62 \pm 8.8 \mathrm{~min}$ to $>1000 \mathrm{~min}$ (Figure 4a). Addition of Q-PEG and spermidine also allowed the clot to remain adhesive under tPA-induced lysis $(1.6 \pm 0.35 \mathrm{kPa}$, compared to $1.8 \pm 0.26 \mathrm{kPa}$ without tPA treatment, Figure $4 \mathrm{~b})$. In contrast, without the FXIIIa-cross-linkable polymer, clots easily detached when treated with tPA, and no adhesion was detected at $90 \mathrm{~min}$. This shows that the FXIIIa-cross-linkable polymer is more resistant to tPA-induced lysis and helps prevent the detachment of clots under fibrinolytic conditions.

\section{CONCLUSIONS}

In summary, this work shows that copolymerizing blood clots with a FXIIIa-cross-linkable synthetic polymer can increase the adhesion of clots in a FXIIIa-dependent manner. This increase in adhesive strength was found both on collagen surfaces and on the subendothelia of excised blood vessels. Together, the data show the effect Q-PEG has on clots is to link blood components to collagen via FXIIIa. The FXIIIa-cross-linkable polymer recovered the adhesive strength of whole blood clots deficient in fibrinogen and maintained the adhesion of plasma clots treated with tPA, two conditions where clots were otherwise non-adhesive.

Within the larger goal of creating advanced hemostatic agents, this work demonstrates that the adhesion of clots to vascular surfaces can be increased with a material besides fibrinogen. This is the first time that the adhesion of plasma-based or whole blood-based clots has been increased with a molecule exogenous to the coagulation system. The specific material used here was chosen for these proof-of-concept experiments because it was already known to cross-link by FXIIIa, not because it met all the criteria for development into a therapeutic. Nevertheless, this discovery-phase work may assist in the conceptualization and design of advanced hemostatic agents that increase the adhesion of clots. Specifically, this concept may enable the design of therapeutics for treating fibrinogen deficiency. Fibrinogen deficiency can occur congenitally due to genetic defects in protein synthesis or its secretion ${ }^{28}$, or it can be acquired, when antibodies are formed against fibrinogen ${ }^{29}$ or when fibrinogen is depleted in trauma-induced coagulopathy (TIC) ${ }^{30}$.

TIC occurs in one in four trauma cases and is associated with a four-fold increase in patient mortality ${ }^{26}$. Fibrinogen deficiency accompanies TIC and infusions of fibrinogen and/or FXIII concentrates are treatments being investigated for this condition ${ }^{6,7}$. Our results show that a FXIIIa-cross-linkable material can recover clot adhesion in human whole blood artificially depleted of fibrinogen, and whole blood from congenitally fibrinogen-deficient mice. In contrast, without the polymer, adhesion was not detected. Hyperfibrinolysis also accompanies TIC. The lysis of plasma clots via tPA quickly renders them non-adhesive. However, our results show that by supplementing plasma with a FXIIIa-cross-linkable material, clots remained adhesive. With further development, this strategy of increasing adhesion with FXIIIa-cross-linkable materials may lead to a synthetic alternative for 
fibrinogen or a complementary additive for treating TIC. However, patients with TIC may also suffer impaired platelet function and acidosis ${ }^{26,30-32}$, and therefore future experiments using blood samples from patients with TIC or in vivo models of TIC would be required for testing the safety and efficacy of this therapeutic strategy.

\title{
Supplementary Material
}

Refer to Web version on PubMed Central for supplementary material.

\section{Acknowledgments}

\author{
Funding Sources
}

This work was funded by Canadian Institutes of Health Research (MOP-119426 and MSH-130166), the Canadian Foundation for Innovation (31928), and the BC Knowledge Development Fund.

The authors thank J. Baylis, M. Lee, and I. Welch for collecting sheep carotid artery tissues and mouse blood. We thank F. Castellino for providing the fibrinogen-deficient mouse strain. We thank S. Novakowski, V. Chan, W. Hur, and N. Mazinani for assisting in collecting blood from human donors.

\section{ABBREVIATIONS}

$\begin{array}{ll}\text { FXIIIa } & \text { coagulation factor XIIIa } \\ \text { Q } & \text { glutamine } \\ \text { PEG } & \text { polyethylene glycol } \\ \text { GPC } & \text { gel permeation chromatography } \\ \text { T101 } & \text { Tetramethyl-2-[(2-oxopropyl)thiol]imidazolium chloride } \\ \text { APTT } & \text { activated partial thromboplastin time } \\ \text { FG } & \text { fibrinogen } \\ \text { FG-def } & \text { fibrinogen-deficient } \\ \text { WB } & \text { whole blood } \\ \text { TIC } & \text { trauma-induced coagulopathy }\end{array}$

\section{References}

1. Ruggeri ZM, Mendolicchio GL. Circ Res. 2007; 100:1673-1685. [PubMed: 17585075]

2. Hart RG, Diener HC, Coutts SB, Easton JD, Granger CB, O’Donnell MJ, Sacco RL, Connolly SJ. Lancet Neurol. 2014; 13:429-438. [PubMed: 24646875]

3. Duarte AP, Coelho JF, Bordado JC, Cidade MT, Gil MH. Prog Polym Sci. 2012; 37:1031-1050.

4. Sierra DH. J Biomater Appl. 1993; 7:309-52. [PubMed: 8473984]

5. Rourke C, Curry N, Khan S, Taylor R, Raza I, Davenport R, Stanworth S, Brohi K. J Thromb Haemostasis. 2012; 10:1342-1351. [PubMed: 22519961]

6. Levy JH, Goodnough LT. Blood. 2015; 125:1387-1393. [PubMed: 25519751]

7. Franchini M, Lippi G. Blood Transfusion. 2012; 10:23-27. [PubMed: 22153684] 
8. Bornikova L, Peyvandi F, Allen G, Bernstein J, Manco-Johnson MJ. J Thromb Haemostasis. 2011; 9:1687-1704. [PubMed: 21711446]

9. Brohi K, Cohen MJ, Ganter MT, Schultz MJ, Levi M, Mackersie RC, Pittet JF. J Trauma: Inj, Infect, Crit Care. 2008; 64:1211-1217.

10. Ball AM, Winstead PS. Pharmacotherapy. 2008; 28:1383-1390. [PubMed: 18956998]

11. Muszbek L, Bereczky Z, Bagoly Z, Komaromi I, Katona E. Physiol Rev. 2011; 91:931-972. [PubMed: 21742792]

12. Mosher DF, Schad PE, Vann JM. J Biol Chem. 1980; 255:1181-1188. [PubMed: 7356656]

13. Dardik R, Shenkman B, Tamarin I, Eskaraev R, Harsfalvi J, Varon D, Inbal A. Thromb Res. 2002; 105:317-323. [PubMed: 12031826]

14. Milleret V, Simona BR, Lienemann PS, Voros J, Ehrbar M. Adv Healthcare Mater. 2014; 3:508514.

15. Lienemann PS, Karlsson M, Sala A, Wischhusen HM, Weber FE, Zimmermann R, Weber W, Lutolf MP, Ehrbar M. Adv Healthcare Mater. 2013; 2:292-296.

16. Mosiewicz KA, Kolb L, van der Vlies AJ, Martino MM, Lienemann PS, Hubbell JA, Ehrbar M, Lutolf MP. Nat Mater. 2013; 12:1072-1078. [PubMed: 24121990]

17. von Maltzahn G, Park JH, Lin KY, Singh N, Schwoppe C, Mesters R, Berdel WE, Ruoslahti E, Sailor MJ, Bhatia SN. Nat Mater. 2011; 10:545-552. [PubMed: 21685903]

18. Sanborn TJ, Messersmith PB, Barron AE. Biomaterials. 2002; 23:2703-2710. [PubMed: 12059019]

19. Yeon JH, Chan KYT, Wong TC, Chan K, Sutherland MR, Ismagilov RF, Pryzdial ELG, Kastrup CJ. Sci Rep. 2015; 5:10274. [PubMed: 25975772]

20. Hokugo A, Takamoto T, Tabata Y. Biomaterials. 2006; 27:61-67. [PubMed: 16000222]

21. Zhao HG, Ma L, Gao CY, Shen JC. J Biomed Mater Res Part B. 2009; 88B:240-249.

22. Andre P, Denis CV, Ware J, Saffaripour S, Hynes RO, Ruggeri ZM, Wagner DD. Blood. 2000; 96:3322-3328. [PubMed: 11071623]

23. Ploplis VA, Wilberding J, McLennan L, Liang Z, Cornelissen I, DeFord ME, Rosen ED, Castellino FJ. Am J Pathol. 2000; 157:703-708. [PubMed: 10980108]

24. Welch BL. Biometrika. 1951; 38:330-336.

25. Nieswandt B, Watson SP. Blood. 2003; 102:449-461. [PubMed: 12649139]

26. Hess JR, Brohi K, Dutton RP, Hauser CJ, Holcomb JB, Kluger Y, Mackway-Jones K, Parr MJ, Rizoli SB, Yukioka T, Hoyt DB, Bouillon B. J Trauma: Inj, Infect, Crit Care. 2008; 65:748-754.

27. Cardenas JC, Matijevic N, Baer LA, Holcomb JB, Cotton BA, Wade CE. Shock. 2014; 41:514521. [PubMed: 24667610]

28. Vu D, Neerman-Arbez M. Thromb Haemostasis. 2007; 5:125-131.

29. Devries A, Rosenberg T, Boss JH, Kochwa S. Am J Med. 1961; 30:486-494. [PubMed: 13720931]

30. Fries D, Martini WZ. Br J Anaesth. 2010; 105:116-121. [PubMed: 20627882]

31. White NJ. Hematology-American Society of Hematology Education Program. 2013:660-663. [PubMed: 24319248]

32. Sorensen B, Fries D. Br J Surg. 2012; 99:40-50. [PubMed: 22441854] 
a

a fibri

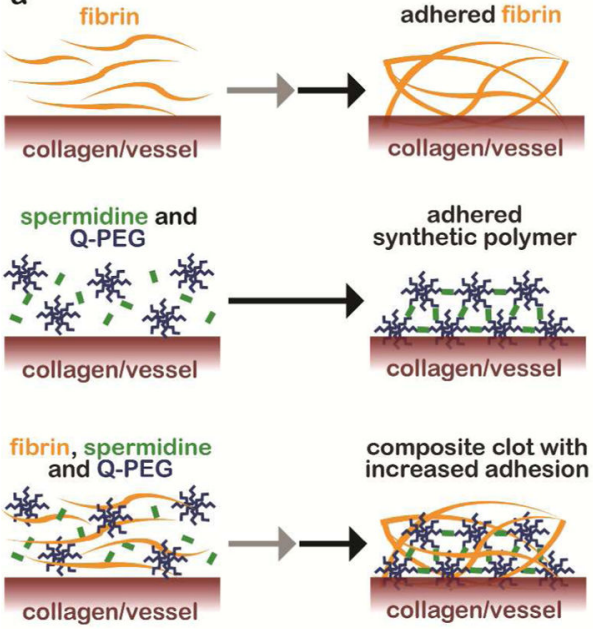

b

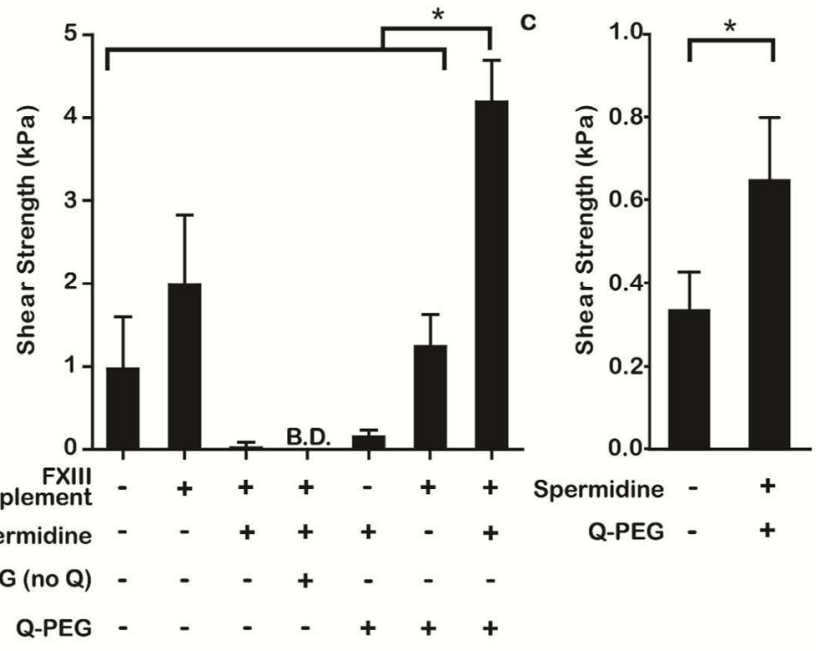

Figure 1. The shear adhesive strength of a normal plasma clot to collagen or the surfaces of excised blood vessels can be increased by adding a FXIIIa-cross-linkable synthetic polymer (a) Schematic showing the self-assembly of fibrin (grey arrow) and the copolymerization of a FXIIIa-cross-linkable polymer (black arrow) increasing adhesion of the resulting clot. (b) Graph shows the maximum shear stress plasma clots withstood before they mechanically failed in a lap-shear adhesion test, with combinations of supplemental FXIII $(0.067 \mathrm{mg} / \mathrm{mL})$, spermidine (1.3 mM), a non-cross-linkable PEG (46 mg/mL) and Q-PEG (46 mg/mL). (c) Graph shows the shear adhesive strength of FXIII-supplemented $(0.13 \mathrm{mg} / \mathrm{mL})$ normal plasma clots formed between two pieces of excised blood vessels. ${ }^{*} \mathrm{p}<0.05$. Data indicate mean \pm SEM, $\mathrm{n}=5-7$. B.D. indicates values below detection $(<0.059 \mathrm{kPa})$. 
a

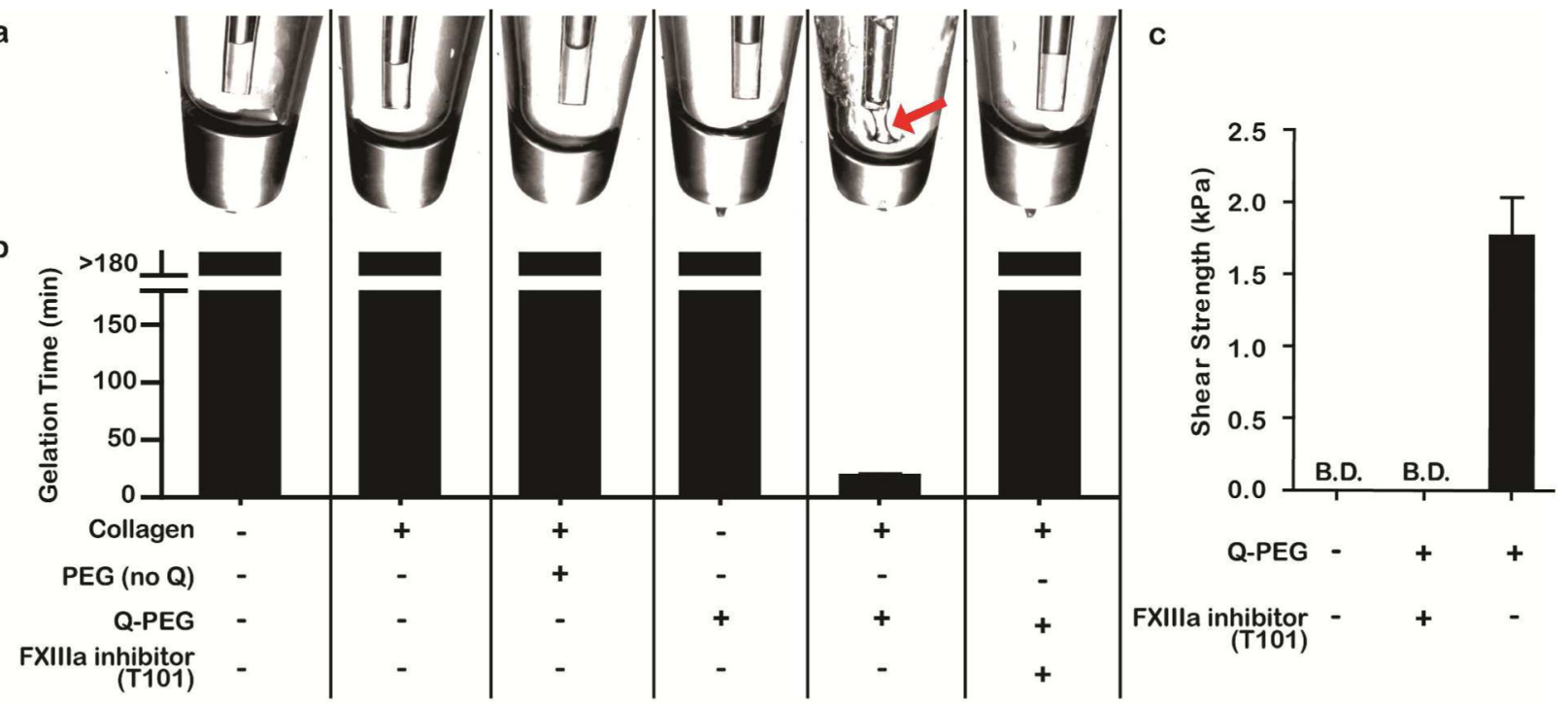

Figure 2. FXIIIa activity controls the binding of Q-PEG to collagen

(a) Images show clot-like gelation of Q-PEG and collagen in FG-def plasma. Gelation can be seen in the image with the red arrow, where the gel became stretched by the micropipette tip. (b) Gelation times of samples in panel a. $n=3$. (c) The shear strength of Q-PEG and spermidine gels formed onto a collagen-coated surface with and without T101. $\mathrm{n}=5$. Data indicate mean \pm SEM. B.D. indicates values below detection $(<0.059 \mathrm{kPa})$. 
a
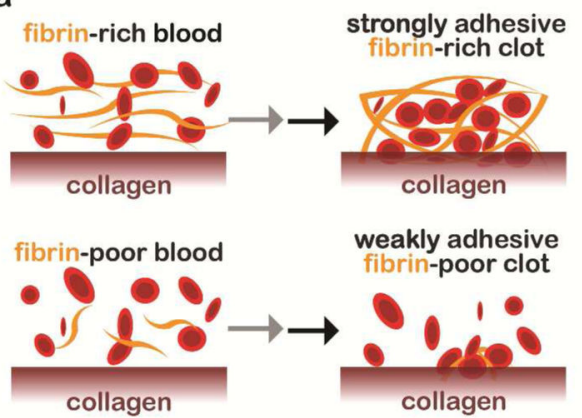

spermidine, Q-PEG
in fibrin-poor blood

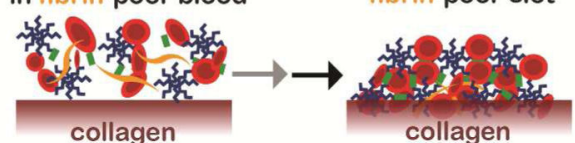

b

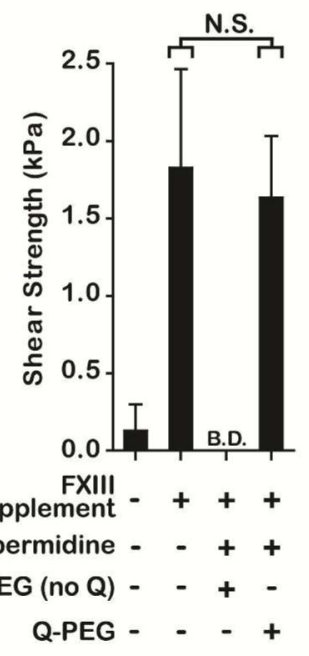

C

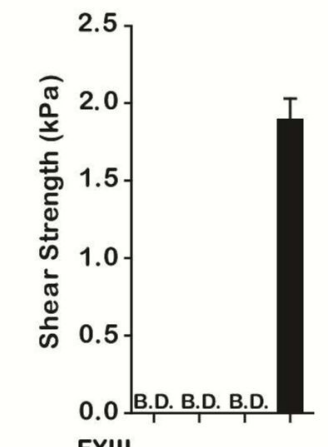

supplement - +++

Spermidine - - + +

PEG (noQ) - - + -

Q-PEG - - - + d

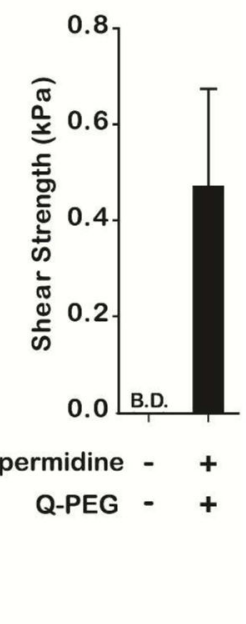

Figure 3. The shear adhesive strength of a FG-def whole blood clot can be increased by adding a FXIIIa-cross-linkable synthetic polymer

(a) Schematic showing a FXIIIa-cross-linkable polymer adhering a whole blood clot to a surface of collagen. (b) Shear strength of fibrinogen-rich human whole blood clots. No significant difference (N.S., $\mathrm{p}>0.05$ ) detected between FXIII-supplemented whole blood with and without Q-PEG and spermidine. (c) Shear strength of FG-def human whole blood clots. (d) Shear strength of clots from whole blood collected from congenitally FG-def mice and supplemented with FXIII $(0.067 \mathrm{mg} / \mathrm{mL})$. Data indicate mean \pm SEM, $n=5$. B.D. indicates data below detection $(<0.059 \mathrm{kPa})$. 
a

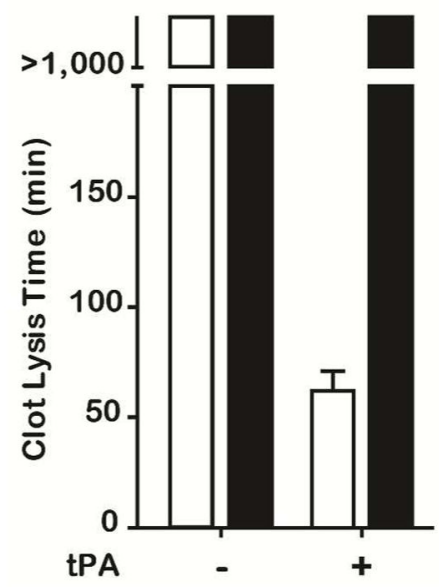

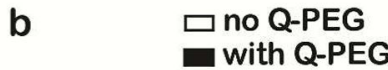

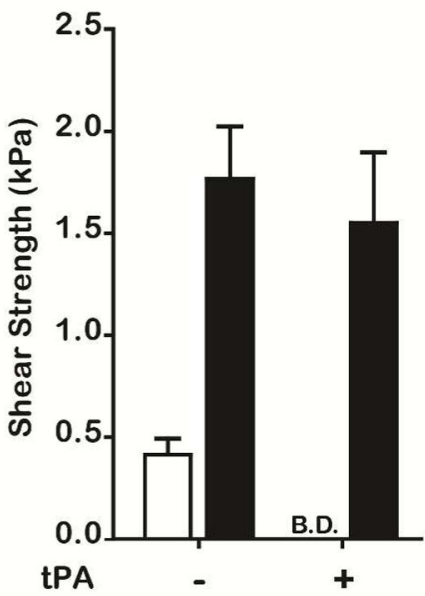

Figure 4.

The shear adhesive strength of a normal plasma clot can be maintained under fibrinolytic conditions by adding a FXIIIa-cross-linkable synthetic polymer. (a) Lysis times of tPAtreated plasma clots with or without Q-PEG. (b) Shear strength of tPA-treated plasma clots at 90 min with or without Q-PEG. Data indicate mean \pm SEM, $n=5-6$. B.D. indicates data below detection $(<0.059 \mathrm{kPa})$. 